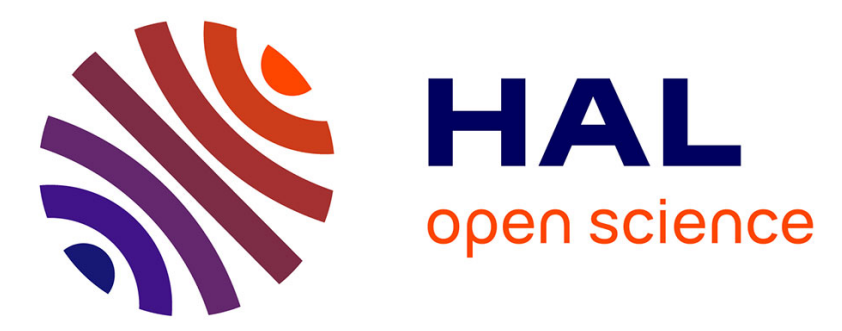

\title{
Le design est-il en passe de devenir une science de gouvernement ? Réflexion sur les espoirs suscités par les sciences du design dans la modernisation de l'État en France (2014-2019) \\ Marie Alauzen, Coline Malivel
}

\section{To cite this version:}

Marie Alauzen, Coline Malivel. Le design est-il en passe de devenir une science de gouvernement ? Réflexion sur les espoirs suscités par les sciences du design dans la modernisation de l'État en France (2014-2019). Sciences du Design, 2020, 12, pp.36-47. 10.3917/sdd.012.0036 . hal-03123467

\section{HAL Id: hal-03123467 \\ https://hal.science/hal-03123467}

Submitted on 27 Jan 2021

HAL is a multi-disciplinary open access archive for the deposit and dissemination of scientific research documents, whether they are published or not. The documents may come from teaching and research institutions in France or abroad, or from public or private research centers.
L'archive ouverte pluridisciplinaire HAL, est destinée au dépôt et à la diffusion de documents scientifiques de niveau recherche, publiés ou non, émanant des établissements d'enseignement et de recherche français ou étrangers, des laboratoires publics ou privés. 


\section{Le design est-il en passe de devenir une science de gouvernement?}

Réflexion sur les espoirs suscités par les sciences du design dans la modernisation de l'État (2014-2018)

\section{Marie Alauzen et Coline Malivel}

Résumé : Constatant le rôle pris ces dernières années en France par le design dans la modernisation de l'État, nous nous demandons si ce dernier ne serait pas en passe de devenir une science de gouvernement au même titre que la statistique, la cartographie, la criminologie, l'archivistique, l'économie politique ou encore les études d'opinion. L'article s'inscrit dans la continuité de réflexions récentes en histoire des sciences du design et de questionnements politiques plus anciens sur les sciences de gouvernement des États modernes. En revenant sur l'incorporation du design par l'administration française de la modernisation de l'État entre 2014 et 2019, il propose une mise en ordre des interventions menées, ainsi que des espoirs et frustrations suscités par l'avènement du design en administration.

Mots clés: sciences de gouvernement, histoire des sciences, modernisation de l'État, sciences du design

Abstract: The article questions the role that design has played in recent years in the modernization of the state in France. We wonder if it is not becoming a science of government in the same way as statistics, cartography, criminology, archival science, political economy or opinion studies did. It is drawing on some recent debates in the history of design sciences and of older political ones on the sciences of government of modern states. Returning to the incorporation of design by the French administration of the modernization of the State between 2014 and 2019, it gives an order to the implementations carried out as well as hopes and frustrations raised by the advent of design in public administrations.

Keywords: sciences of government, social studies of science, modernization of the State, science of design 
Le 27 février 2019, le comité de pilotage du Plan d'investissement d'avenir (PIA) a approuvé le financement de « designers d’intérêt général » recrutés pour apporter leur expertise à des projets soumis par des administrations ${ }^{1}$. Ce programme de la loi de finances visant à doter certains secteurs réputés stratégiques de crédits supplémentaires a conféré une large visibilité à une tendance récente de la modernisation de l'État. Depuis 2014, en France, le design est en effet devenu une expertise incontournable dans l'activité coordonnée de réforme de l'État et participe à façonner de nouvelles « formes d'innovation publique » (Coblence et Pallez, 2015 ; Coblence et Vivant, 2017 ; Weller et Pallez, 2017).

La réforme ou modernisation de l'État désigne l'activité coordonnée de transformation de l'État que mène une administration dédiée : entre 2008 et 2012, cette administration prenait le nom de Direction générale de la modernisation de l'État (DGME), puis, de 2012 à 2017, de Secrétariat général pour la modernisation de l'action publique (SGMAP), et, depuis, de Direction interministérielle de la transformation de l'action publique (DITP), d'un côté, et la Direction interministérielle du numérique et du système d'information et de communication de l'État (DINSIC) de l'autre. Le contenu de cette activité administrative est hautement politique et varie selon les arrangements institutionnels, c'est-à-dire les dynamiques d'action collective que conduisent certains acteurs en concurrence historiquement, la Direction générale des collectivités locales, la Direction du budget et la Direction générale de la fonction publique (Bezes, 2009). Ces acteurs ne mobilisent pas un répertoire de techniques et de savoirs homogènes et stabilisés, mais agencent des moyens d'agir en fonction des problèmes désignés, des diagnostics du moment, des théories implicites et des inclinations politiques.

Au cours de la sulfureuse Révision générale des politiques publiques (RGPP) conduite par la DGME entre 2008 et 2012, moderniser l'État signifiait procéder à des fusions, des restructurations dans les ministères et les services déconcentrés de l'État (Bezes, 2012; Bezes et Le Lidec, 2016), mais aussi intervenir sur les pratiques les plus quotidiennes et les modes d'interaction des agents, sur leurs instruments de gestion et l'organisation du travail (Gélédan, 2012). Pour ce faire, la DGME a introduit des experts allogènes aux grands corps et aux sociologues des organisations : des consultants et des auditeurs aptes à faire le lien entre la vision budgétaire de l'État instillée par les gouvernements Fillon et les modes de transformation des organisations publiques (Bezes, 2012; Gélédan, 2019). Les consultants frappaient à la porte des administrations avec leurs savoirs managériaux ajustés sur les standards des cabinets de conseil anglo-saxons. Le propos des méthodes de productivité - dont le lean management, les reportings hebdomadaires et le pullulement de supports PowerPoint - était, entre autres, de

${ }^{1}$ Nous remercions Marine Gout ainsi que les évaluateurs pour leurs lectures attentives. Les propos formulés dans cet article n'engagent que leurs autrices. 
« hâter la mue des bureaucrates en managers » (Gélédan, 2012, paragr. 2).

Puis, avec l'élection du gouvernement socialiste en 2012, la réforme de l'État a pris une tout autre coloration. Le cahier des charges a changé : il fallait désormais simplifier le «travail administratif des usagers » (ou paperasserie), valoriser les initiatives portées par les agents publics, créer des infrastructures numériques communes à l'ensemble des ministères et expérimenter de nouvelles façons de collaborer. Le maitre mot était l'innovation. Les modernisateurs ont eu recours à de nouvelles expertises issues des sciences sociales (notamment les sciences participatives et la sociologie du travail administratif), de l'économie comportementale, de l'informatique et du design (Gélédan, 2016). L’article propose de se concentrer sur l'avènement de ce dernier domaine d'expertise dans la modernisation de l'État et d'éclairer la façon dont, en quelques courtes années, le design est devenu une médiation légitime de l'intérêt général et un investissement d'avenir. Aussi, en marge des réflexions épistémologiques qui sondent le rôle que pourrait ou devrait avoir le design dans les politiques publiques, nous nous intéresserons aux actes de design tels qu'ils se font depuis quelques années dans les administrations françaises.

Le développement qui suit s'appuie sur deux types de sources assemblées et analysées conjointement par les deux autrices. Les premiers matériaux sont ethnographiques et ont été recueillis dans le cadre d'une thèse menée au SGMAP, entre 2014 et 2017, et plus particulièrement auprès des équipes responsables de la promotion des méthodes de design dans les projets de transformation de l'État (notes de terrain produites au jour le jour, collecte documentaire de l'activité quotidienne et sélection d'archives numériques)². Les seconds matériaux mêlent un savoir expérientiel et des archives personnelles recueillies en tant que designeuse prestataire du SGMAP (été 2015), puis designeuse intégrée au SGMAP (2015-2017) et, à nouveau (depuis 2017), intervenante ponctuelle, sollicitée pour son expertise sur la modernisation de l'État par le design.

Après clarification de ce que nous entendons par «sciences de gouvernement », nous analyserons les premiers entrecroisements du design et de la réforme de l'État en France. Nous examinerons ensuite, dans la chair des projets de modernisation, comment le design est devenu cette expertise indispensable.

1. Des sciences historiques de gouvernement au design, en passant par la modernisation de l'État

\subsection{Quelques précisions sur les sciences de gouvernement}

${ }^{2}$ Entre 2014 et 2017, la thèse a bénéficié d'une convention pour le financement d'une recherche doctorale entre le SGMAP et Mines ParisTech. 
Interpellées par l'irruption du design dans la modernisation de l'État, nous nous demandions en exergue de cet article s'il n'était pas en train de devenir une science de gouvernement. Les sciences de gouvernement, autrement appelées sciences d'État, désignent un domaine d'expertise porté par un corps de métier attitré qui vise à saturer de sa présence et de son savoir-faire la manière de gouverner. L’invention de l'État moderne s'est en effet accompagnée «d'un intense travail théorique de justification, d'explication dont émergent quelques grands noms, toujours cités (Machiavel, Bodin, Hobbes, Locke, pour la tradition européenne) » (Bourdieu, Christin et Will, 2000, p. 5). Cette philosophie politique a offert «aux princes de nouveaux instruments conceptuels de gouvernement et de légitimation sous la forme d'innombrables publications, de thèses, de disputes, de recueils de lois, mais aussi d'interventions directes d'experts au sein d'instances de pouvoir » (Bourdieu, Christin et Will, 2000, p. 5). Des administrateurs experts consacrés à l'écrit royal (Ferrer-Bartomeu, 2017) aux protoarchivistes compilant les actes à valeur perpétuelle scellés de cire verte (Potin, 2000), ces sciences parfois rassemblées sous le titre général de sciences camérales ont contribué à la forme bureaucratique de l'État moderne que nous connaissons aujourd'hui (Hood, 2000).

Outre les sciences de l'État mises au jour par la tradition bourdieusienne, l'histoire des sciences (Shapin et Schaffer, 1993) et les sciences politiques inspirées par les travaux de Michel Foucault sur la gouvernementalité (Laborier, Audren, Napoli et Vogel, 2011) ont attiré l'attention sur l'avènement d'un régime d'objectivité propre à certains savoirs sécularisés de gouvernement. À partir du XVIII ${ }^{\mathrm{e}}$ siècle, en plus d'établir les doctrines du droit, de l'économie politique et de la police, les sciences de gouvernement ont raffiné leurs instruments afin d'apporter aux administrations une aide à la décision dans les domaines des forêts (Scott, 1998), des mines (Vogel, 2011), de la démographie (Overath, 2011), de la gestion des grèves (Tanguy, 2011 ), de la statistique (Bardet, 2003 ; Behrisch, 2011 ; Retière, 2003) ou encore de la cartographie (Ingold, 2011 ; Le Bourhis, 2003). Revendiquant avec plus ou moins de succès une scientificité propre (Ihl, 2006), ces domaines ont accompagné la plupart des grands projets administratifs et politiques: de la colonisation (Dimier, 2003; Mitchell, 1988) à la lutte antiterroriste (Linhardt et Moreau de Bellaing, 2017), en passant par le tournant participatif des politiques publiques (Blondiaux, 1998 ; Jeannot, 2006).

À un moment où, en France, le design est aussi un champ académique en cours d'institutionnalisation (Vial, 2015), où le marché est sur le point de se métamorphoser et où le recours aux designeurs se fait de plus en plus évident dans les politiques publiques, cette généalogie permet d'interroger à nouveaux frais les techniques contemporaines de gouvernement. 


\subsection{Rencontre politique et marchande du design et de la modernisation de l'État}

L'interface entre le design et la réforme de l'État a d'abord été de nature politique. Bénéficiant de la dynamique engendrée par la RGPP sur la modernisation de l'État et d'un phénomène transnational (Weller et Pallez, 2017), La 27e Région a été créée en 2008 par l'Association des régions de France pour promouvoir l'innovation dans les collectivités locales, grâce aux compétences de designeurs de services (social design). Au moment du changement de gouvernement et de l'effacement de la DGME au profit du SGMAP en 2012, les méthodes indiquées par La 27e Région, déclarées attentives aux pratiques des usagers, des agents et à l'écologie des administrations, sont apparues comme une précieuse solution de remplacement des savoirs néomanagériaux des consultants. La préfiguration du caractère alternatif des méthodes d'innovation de l'association a été favorisée par l'entremise de son président historique, Christian Paul, député membre de la nouvelle majorité socialiste et conseiller régional de Bourgogne. Proches de Marylise Lebranchu, ministre de la Réforme de l'État, de la Décentralisation et de la Fonction publique du gouvernement Ayrault, Christian Paul et le directeur de l'association ont réalisé un travail politique permettant à La 27e Région de diversifier ses sources de financement (Union européenne, Caisse des dépôts et des consignations, Ville de Paris) et de percevoir une enveloppe du SGMAP. Outre le financement qui a permis à l'association d'inaugurer SuperPublic en 2014, un espace de travail consacré à la transformation de l'action publique dans le $\mathrm{XI}^{\mathrm{e}}$ arrondissement de Paris, le SGMAP et La 27e Région ont concrétisé des projets ensemble. Une designeuse de l'association a alors intégré la mission méthodes d'écoute et d'innovation avec les usagers pour initier les agents du SGMAP aux arts de faire du design, et La 27e Région a conduit, pour le compte du SGMAP, une « résidence » sur l'action sociale communale, à Cluny, durant le premier semestre 2014 (Chevrot, 2018 ; Coblence et Pallez, 2015).

L'introduction de La 27e Région dans l'activité de réforme de l'État dont le SGMAP avait la responsabilité a précipité la formation d'un marché. Dans le sillage de l'association, des agences de « design d'intérêt général » ont été créées. Certaines étaient directement hébergées à SuperPublic. Les agences historiques se sont restructurées pour répondre aux marchés publics et les gros cabinets de conseil, bénéficiaires des principaux marchés, ont commencé à racheter ou fonder leurs agences : depuis 2013, Fjord appartient à Accenture, La Javaness est une branche d'Eurogroup Consulting, etc. Le SGMAP a publié un premier marché consacré au design de services publics en 2014, puis le ministère de l'Éducation nationale et quelques collectivités territoriales ont suivi le mouvement. Depuis 2015, tous les marchés publics de modernisation requièrent que l'offre commerciale soit accompagnée de prestations de design. 
Il est demandé au prestataire d'apporter son expertise en matière de conception de services, d'organisation, d'outils ou de plateformes numériques en se basant sur l'expérience des usagers. Les techniques d'animation et de conception de type «design thinking» seront privilégiées et pourront être associées à d'autres modalités d'accompagnement ${ }^{3}[\ldots]$ [Le lean management] pourra nécessiter de mettre en œuvre une démarche de type design de service ${ }^{4}$.

L'attraction du design dans la fabrique des politiques publiques est devenue telle que l'École nationale d'administration (ÉNA) et l'École nationale supérieure de création industrielle (ÉNSCI) ont inauguré, en 2017, la Chaire innovation publique, qui a, par exemple, utilisé les méthodes du design pour réformer un objet administratif historique, le rapport de la Cour des comptes. Enfin, en 2019, le PIA a permis l'embauche de 21 « designeurs d'intérêt général » répartis en 17 projets de refonte de l'expérience utilisateur (UX design) des interfaces administratives.

Ces opérations politiques et économiques signalent l'émergence d'une certaine politique du design - par ailleurs débattue dans le champ académique (Berger, 2015) — et attirent l'attention sur les spécificités de l'ambition modernisatrice. Voyons maintenant plus précisément ce que le design fait à l'État en réformes.

\section{Le design : un savoir indispensable à la modernisation de l'État}

\subsection{Classification des interventions (2014-2019)}

Des premières immixtions du design de services publics et de l'activité coordonnée de modernisation de l'État en 2014 au lancement du PIA en 2019, nous distinguons trois types de manifestations du design qui l'apparentent à une science de gouvernement: l'établissement d'infrastructures, la sollicitation pour renouveler les arts de faire la modernisation et l'invitation à rejoindre des projets de modernisation.

Nous l'avons lu : incorporer le design à la modernisation de l'État n'est en rien une opération de l'esprit. Il a d'abord fallu tisser un partenariat avec La 27e Région, intégrer des designeuses dans les équipes du SGMAP (en 2014, puis entre 2015 et 2017), libeller des marchés publics structurant le recours aux agences

\footnotetext{
3 SGMAP, Gahier des clauses techniques particulières, assistance à la conception et à la mise en œuvre opérationnelle de projets de transformation de l'action publique, 2015, p. 12.

4 Ibid., p. 15.
} 
(2014-2018 et 2019-2023), puis faire valoir le design comme investissement d'avenir (2019). Toutes ces opérations juridiques et économiques sont éminemment matérielles et relationnelles : elles supposent une documentation et des recherches préalables à la rédaction d'argumentaires, de fiches de poste et à la mise à disposition de logiciels, de matériel informatique ou d'articles de bureau. De fil en aiguille, elles ont composé l’infrastructure du design dans la réforme de l’État.

«Vous allez nous changer» était la ritournelle entendue par les designeuses qui ont rejoint les rangs des modernisateurs. Ces derniers nourrissaient de vives attentes quant aux savoir-faire des nouvelles venues et les sollicitèrent pour rénover les méthodes du SGMAP : stylisation de la réunion de service, inauguration d'un «lab», aspiration au compromis grâce au design thinking; le tout dans la bonne humeur. Il fallait non seulement regonfler la modernisation de l'État, lui redonner une forme désirable après les critiques de la RGPP, mais aussi formaliser les méthodes de travail dans un «kit» dont pourraient s'emparer les acteurs de la modernisation et des autres administrations ${ }^{5}$.

Enfin, grâce à la stabilisation progressive de cette infrastructure bureaucratique et à l'acculturation du SGMAP, la modernisation de l'État a procédé à un certain nombre d'actes de design. Nous en dénombrons neuf pour la période 2014-2017, répartis entre design de service et design visuel. Parmi eux, la plupart étaient davantage de l'ordre du dépannage, de la réparation ou de l'embellissement graphique que de la conception au sens strict. Pour en prendre la mesure, prenons l'exemple du gigantesque programme «Simplification du parcours administratif hospitalier du patient et numérisation des informations échangées » (SIMPHONIE) pour lequel la Direction générale de l'offre de soins (DGOS) a sollicité le SGMAP en 2014.

Près de la moitié du « reste à charge du patient » (c'est-à-dire le montant restant à payer par le bénéficiaire du soin après la prise en compte de ses droits d'assurance maladie obligatoire et complémentaire) n'est jamais recouvert par l'hôpital. À la sortie de l'hôpital, les soignants n'indiquent pas nécessairement aux patients de passer par la caisse et la majorité des «factures » ${ }^{6}$, bien que d'un montant faible pour le patient (17,2€ en moyenne), ne sont jamais honorées. Dans un contexte de recherche d'efficience et de sécurisation des recettes caractérisée par la multiplication des exercices de connaissance des coûts (Alauzen et al, 2020),

${ }^{5}$ Se reporter à «Comment faire, la boîte à outils de l'innovation publique », qui propose de faire découvrir le design public en mettant à disposition une méthode en neuf étapes et des outils de conception. Repéré à : comment-faire.modernisation.gouv.fr/.

${ }^{6}$ Pour comprendre l'enjeu de la requalification de l'avis des sommes à payer comme une facture administrative, se reporter à l'article repéré à : modernisation.gouv.fr/sinspirer-pourtransformer/lean-et-nudge-associes-au-benefice-des-patients-et-des-hopitaux. 
la DGOS a donné pour mission au SGMAP d'aider les établissements de santé à rénover la chaîne de facturation, à mieux recouvrir le paiement des actes de soin et à formaliser les parcours administratifs dans les établissements. Après la construction de parcours suivant des méthodes bien ancrées au SGMAP (Gélédan, 2016), la direction générale des finances publiques a établi une première maquette standardisée de l'« avis des sommes à payer », soit la facture que l'hôpital adresse au bénéficiaire des soins (Fig. 1).

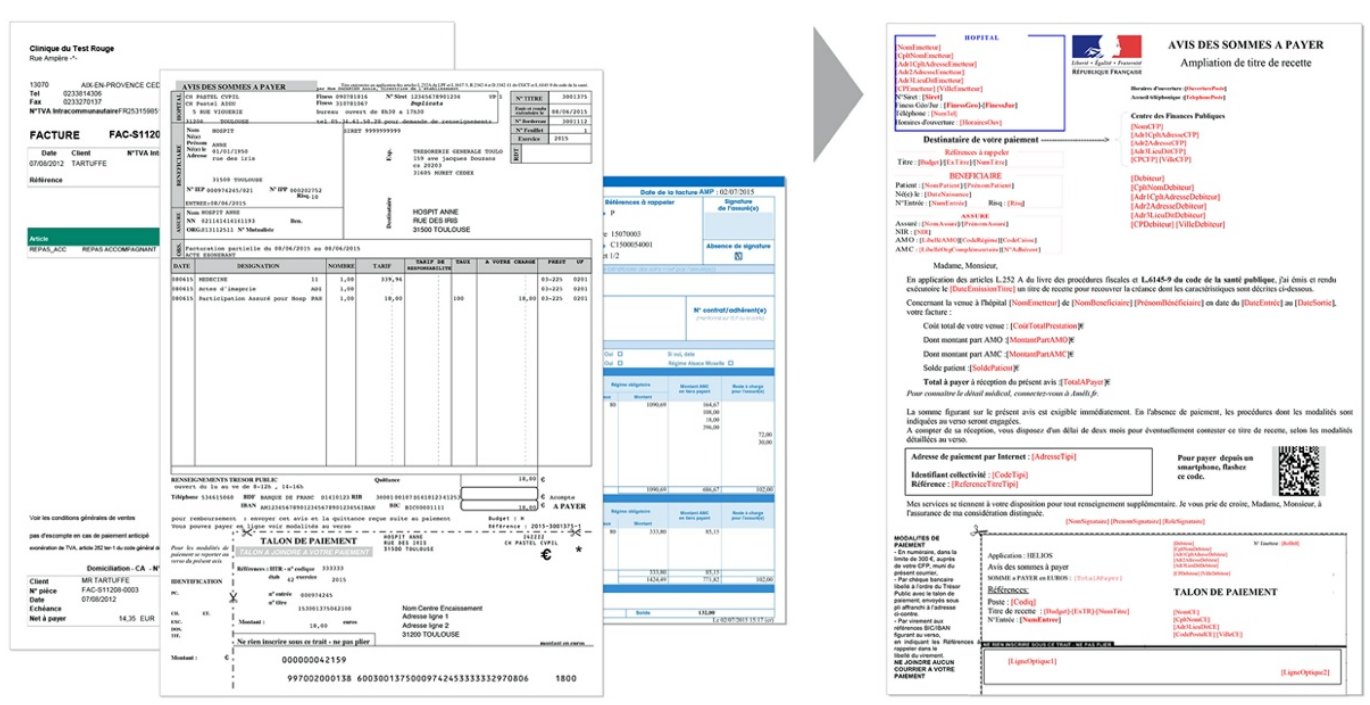

Figure 1. Des exemples d'avis qu'envoyaient les hôpitaux, au modèle standardisé conçu par la Direction générale des finances publiques

En parallèle, l'équipe nudge avait été sollicitée pour diagnostiquer le comportement des patients et pour tester des incitations au paiement de leur séjour. Une partie de leur attention se porta sur le design de l'avis des sommes à payer. À partir des leviers comportementaux identifiés lors d'entretiens (moralité de l'acquittement, pédagogie sur le règlement, culpabilité des mauvais payeurs, etc.), il s'agissait d'ajuster l'avis préalablement lissé en intervenant sur la lisibilité de l'action attendue par l'émetteur : s'acquitter de sa dette (Fig. 2).
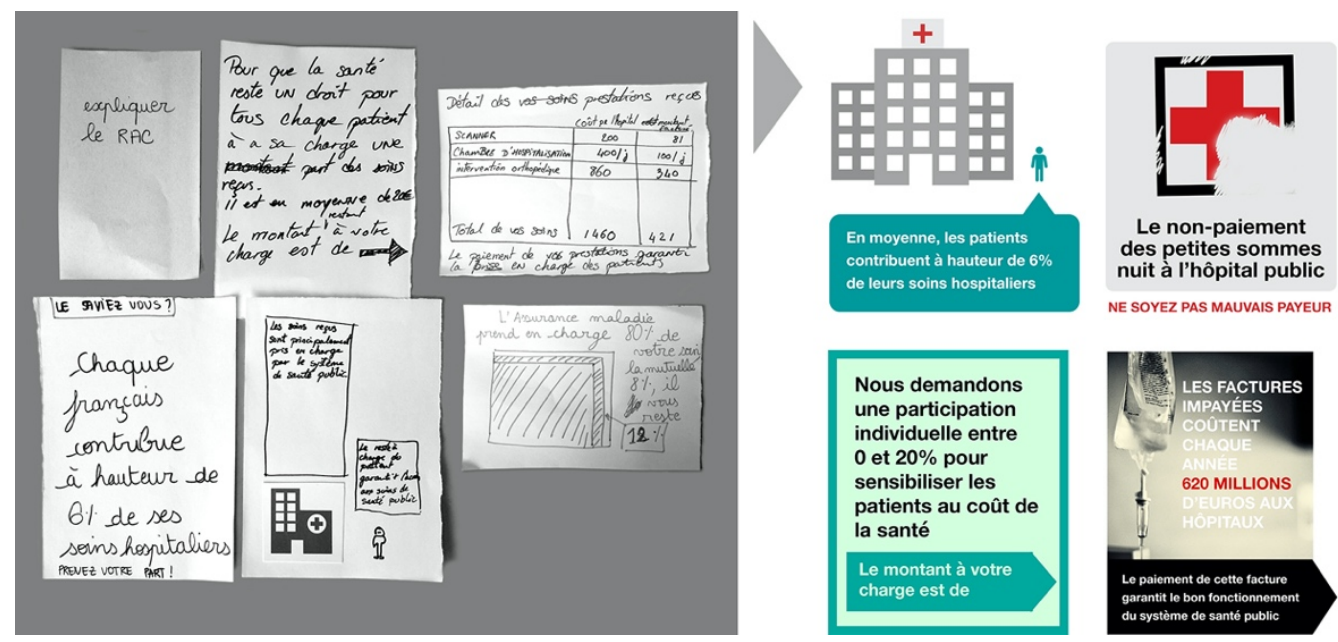
Figure 2. Travail de conception des nudges, avec les parties prenantes, à partir des leviers comportementaux identifiés

Les prototypes des nudges ont ensuite été soumis à un atelier de co-design avec les parties prenantes des différentes administrations avant d'être expérimentés dans les établissements pilotes (Fig. 3).
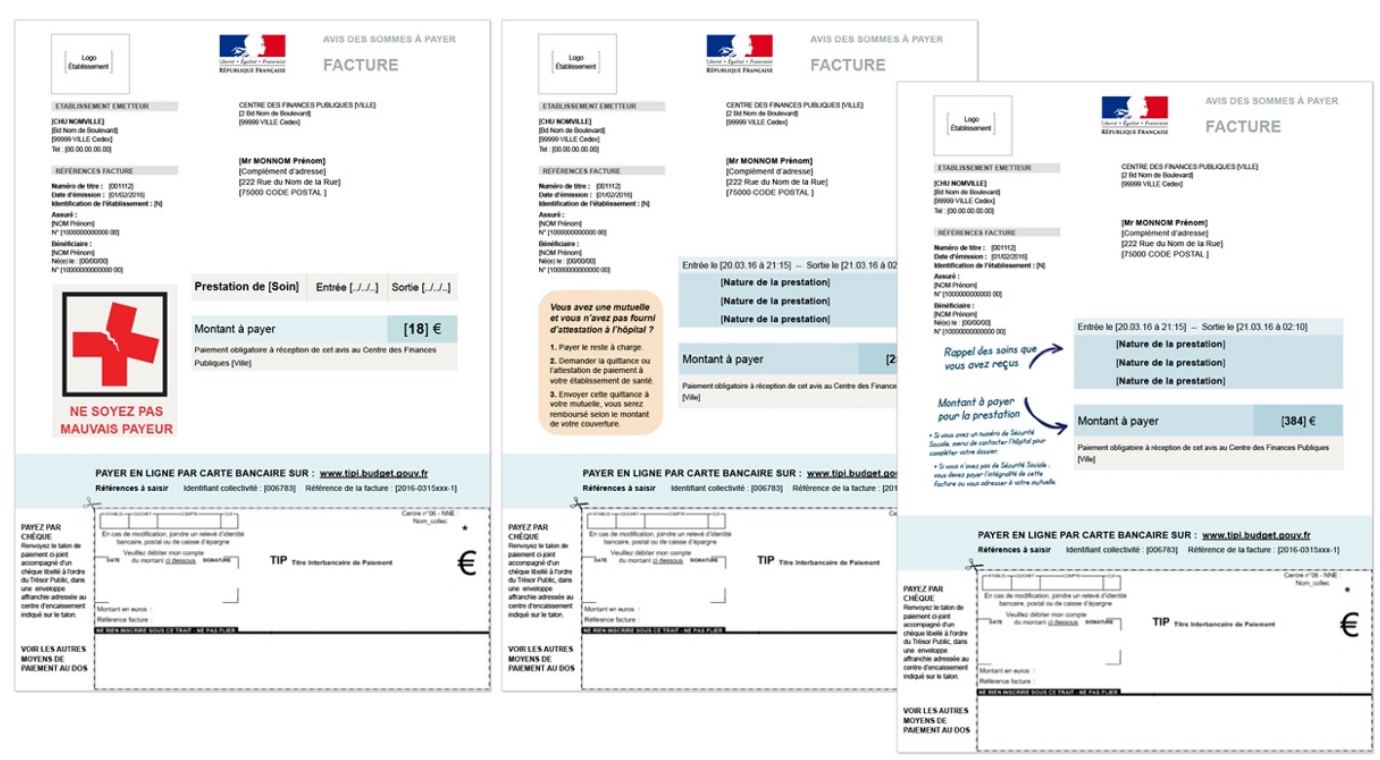

Figure 3. Maquette des trois factures expérimentées dans les établissements pilotes en 2017

Cet acte de design, greffé dans un programme de transformation, constitue une micro-opération de rhabillage de la chaîne de facturation, une utile réfection d'un document administratif, et a fait du design une expertise complémentaire des sciences comportementales, du lean management, des études du coût marginal et des constructions de parcours avant d'intégrer un processus plus classique d'évaluation et de déploiement.

Ainsi, en quelques années, le design s'est invité au cour de l'exercice de modernisation de l'État et est devenu, à sa manière, une science de gouvernement. Quels sont les multiples effets sur l'État de ses capacités émergentes? Pour traquer les actions du design dans la transformation coordonnée de l'État sans y rechercher un impact absolu et prédéterminé, nous suggérons de prêter une attention soutenue aux espoirs et frustrations suscités par ces interventions.

\subsection{Espoirs et frustrations, revue des actes de design en administration (2014-2019)}


Les espoirs portés par le design sont d'ordres différents pour les modernisateurs (DGME, SGMAP, DITP, DINSIC, DINUM), les administrations bénéficiaires des missions et les designeurs eux-mêmes. Nous avons indiqué que la première attente des modernisateurs de l'État était de renouveler leurs savoir-faire à un moment où les outils managériaux promus au temps de la RGPP avaient été vilipendés (Bezes et Le Lidec, 2016 ; Gélédan, 2012). Par ailleurs, l'ethnographie des usages recommandée en amont du travail de conception par les designeurs ne constituait pas une critique véhémente de ce qui se faisait jusqu'alors, mais un affinement de l'approche par l'usager de la réforme de l'État — soit des technologies de mesure de la complexité ressentie par les usagers des services publics et de consultation des publics sur les priorités de la simplification administrative (Gélédan, 2016). Ici, le recours aux usagers, lors d' « immersions sur le terrain » ou de «tests utilisateurs", faisait partie d'une méthodologie expérimentale, inhérente au design et focalisée sur les comportements, la distinguant nettement des autres sciences de gouvernement. En ce sens, l'attelage du design et de la modernisation de l'État paraissait d'autant plus souhaitable qu'elle constituait un objet de projection de fantasmes pour la hiérarchie intermédiaire et les chefs de projets.

Au SGMAP comme dans les administrations commanditaires des projets, le recours au design a soulevé un enthousiasme certain. Les interventions étaient palpables, car les prototypes étaient laissés sur place, les propositions commerciales semblaient peu onéreuses comparativement aux tarifs des cabinets de conseil traditionnels et, contrairement aux projets de refonte d'organisations qui s'étendaient sur plusieurs mois et exigeaient de nombreux comités de pilotage, les interventions des designeurs paraissaient souples et rapides. La séduction était si forte que le design a cristallisé des croyances quasi magiques. «On co-design (sic) directement les politiques publiques avec les usagers », " on propose des services de plus en plus intuitifs », " on accélère n’importe quel projet en animant des ateliers qui bousculent les codes de l'administration», entendait-on dans les couloirs $^{7}$. Autrement dit, le design venait autoriser certains agents à faire, du moins à tester, à incarner des stratégies dans des artefacts; le tout dans une atmosphère contrastant avec les habitudes bureaucratiques. En outre, pour les administrations bénéficiaires, l'horizon des attentes était souvent celui d'un moindre contact avec des usagers qu'il fallait autonomiser grâce à de nouvelles interfaces ou, du moins, celui de limiter le travail de réparation, omniprésent dans la relation de service et jusqu'alors perçu comme incompressible (Weller, 1999).

Enfin, du côté des designeurs qui ont accédé à la commande publique et aux projets de modernisation, l'administration est devenue un territoire à conquérir. Après les consultants en stratégie des organisations de la RGPP (Gélédan, 2019) et les groupes historiques d'acteurs en concurrence pour établir

7 Notes de terrain au SGMAP, 23 novembre 2017. 
le sens de la réforme de l'État (Bezes, 2009), les designeurs se sont positionnés pour atteindre les objets de l'intervention étatique: rapports et autres médiations numériques de la relation administrative, dossiers, bulletins d'information, mais aussi espaces d'accueil des publics. Ils ont déployé des tactiques et des stratégies de pouvoir, rêvant de contrôler des territoires et d'organiser des domaines d'expertise incontestés. Une designeuse a explicité cette tension à la fin de son contrat : «si j'avais voulu aller plus loin, j'aurais dû faire de la politique ${ }^{8}$ ».

Toutefois, ce mouvement d'espoir a également fait surgir un certain nombre de frustrations. La première d'entre elles était partagée au SGMAP, dans les administrations bénéficiaires des missions de design et par les designeurs euxmêmes; elle était le corollaire des croyances magiques et de la légèreté mentionnées plus haut. Tout le monde attendait du design des exploits, une transformation fabuleuse des problèmes historiques de l'administration: la complexité de certaines procédures ici, l'insatisfaction des usagers des services publics là, le manque de personnel plus loin. Or, les réparations indiquées par les designeurs ressemblaient souvent à des embellissements graphiques ou des rustines et laissèrent un goût amer à certains agents. À l'issue d'une mission d'immersion prototypage visant à réformer l'accueil des agences de l'Assurance vieillesse, l'un d'eux soupira : «C'est joli, mais vous avez réinventé ce qu'on faisait déjà depuis des années. En venant une semaine sur place, c'est normal, mais bon, c'est pas très valorisant pour nous ${ }^{9} »$. De leur côté, les designeurs ont pu être exaspérés du contentement des maquettes. «On n'a pas de budget, on a créé des rêves... et donc il n'y a que les prototypes qui restent », murmura l'une d'elles ${ }^{10}$. Le design semblait peiner à sortir de ses propres expérimentations, souvent faute d'appui pour établir la solidité des faits.

\section{Conclusion}

Le design est-il devenu une science de gouvernement? Les récents mouvements de la modernisation de l'État nous amènent à avancer une réponse positive. Qu'il soit visuel, de services ou d'interface numérique, le design a bien accompagné l'extension des interventions de l'État, mettant en forme une véritable ingénierie politique de la réinvention des liens entre l'État et les citoyens. Ceci étant dit, il reste encore à spécifier le genre de science de gouvernement que serait le design et à préciser ce que cette nouvelle qualification ouvre comme espace de réflexivité pour la discipline.

\footnotetext{
${ }^{8}$ Notes de terrain au SGMAP, 18 janvier 2017.

${ }^{9}$ Notes de terrain au SGMAP, 6 décembre 2014.

10 Notes de terrain au SGMAP, 21 mai 2019.
} 
Les travaux sur les sciences de gouvernement ont montré que le terme de « science » est moins un constat qu'une manière de remettre en question le rôle politique des savoirs institutionnalisés, notamment à partir de leurs manières d'établir des faits (Ihl, Kaluszynski et Pollet, 2003 ; Ihl, 2006). En matière de design de services publics, l'établissement des faits mêlait à des hypothèses de l'observation des comportements et des méthodes de provocation empruntées aux sciences sociales, et se réclamait de l'expérimentation. Toutefois, dans chacune des manifestations repérées, le design de services publics a toujours été combiné à d'autres expertises et façons de faire: l'informatisation, l'économie comportementale, le lean management, etc. Il ne régnait pas sans partage sur le territoire de l'État en réformes, mais s'éprouvait quotidiennement dans des heurts, des accommodements et des compromis. D'où, peut-être, un certain nombre de frustrations. Cet entremêlement rend complexe l'évaluation du régime d'objectivité propre à ce design et appelle de nouveaux développements empiriques; d'une part, sur les conditions d'émergence et la fabrication par les designeurs de ces manières d'incarner l'intérêt général et, d'autre part, sur le façonnement d'un problème public comme champ d'intervention du design.

Pour finir, penser le design comme l'une des sciences contemporaines du gouvernement permet de l'inscrire, tant comme spécialité académique que pratique professionnelle, dans le champ des sciences du politique. Cet ancrage invite à interroger collectivement les situations d'enrôlement en politique dont il est le sujet et l'objet, ainsi qu'à examiner son rôle d'aide à la décision et les critiques qui lui sont adressées à l'aune d'une sociohistoire des arts de gouverner.

\section{Références}

Alauzen, M., Muniesa, F. \& Violle, A. (2020). Exercising knowledge of costs: behavioural politics of economic restraint in French public service reform. French Politics. doi : 10.1057/s41253-020-00131-9

Bardet, F. (2003). De la territorialité des sciences de gouvernement. L'installation des directions régionales de la Statistique. Dans Ihl, O., Kaluszynski, M. \& Pollet, G. (dir.), Les Sciences de gouvernement (p. 181-193). Paris : Economica.

Behrisch, L. (2011). «Des chiffres politiques». La statistique, dispositif politique et activité pratique au XVIII siècle. Dans Laborier, P., Audren, F., Napoli, P. \& Vogel, J. (dir.), Les Sciences camérales: activités pratiques et histoire des dispositifs publics (p. 509-537). Paris : Presses universitaires de France.

Berger, E. (2015). Le designer, un praticien politique mais pas politicien. Pour une éthique dans la pratique. Dans Bertrand, G. \& Favard, M. (dir), Pö̈́tiques du design, Conception et politique (p. 57-75). Paris : L'Harmattan.

Bezes, P. (2009). Réinventer l'État. Les réformes de l'administration française (1962-2008). Paris : Presses universitaires de France. 
Bezes, P. (2012). État, experts et savoirs néo-managériaux. Les producteurs et diffuseurs du New Public Management en France depuis les années 1970. Actes de la recherche en sciences sociales, 3 (193), 16-97.

Bezes, P. \& Le Lidec, P. (2016). Politiques de la fusion. Revue française de science politique, $66(3-4), 507-541$.

Blondiaux, L. (1998). La Fabrique de l'opinion. Une histoire sociale des sondages. Paris : Le Seuil.

Bourdieu, P., Christin, O. \& Will. P.-É. (2000). Sur la science de l'État. Actes de la recherche en sciences sociales, (133), 3-11.

Chevrot, B. (2018). De l'isolement social aux territoires sociables. Recherche impliquée sur l'intervention sociale en milieu rural, à l'épreuve de l'innovation territoriale. Thèse de sociologie, Université Lumière Lyon 2.

Coblence, E. \& Pallez, F. (2015). Nouvelles formes d'innovation publique: L'administration saisie par le design. Revue française de gestion, 251 (6), 97-114.

Coblence, E. \& Vivant, E. (2017). Le design est-il soluble dans l'administration ? Trois trajectoires d'institutionnalisation de l'innovation publique. Sciences du Design, 5 (1), 5268.

Dimier, V. (2003). La « science » coloniale en Angleterre et en France. Une arme dans les relations internationales. Dans Ihl, O., Kaluszynski, M. \& Pollet, G. (dir.), Les Sciences de gouvernement (p. 59-85). Paris : Economica.

Ferrer-Bartomeu, J. (2017). L'État à la lettre. La mise en circulation de l'information politique et administrative dans les arcanes du pouvoir (Royaume de France, 15701610), Cahiers d'histoire. Revue d'histoire critique, (134), 41-63.

Gélédan, F. (2012). Le management au secours de services publics? Modernisation de l'État et régimes de domination à l'heure de la Révision générale des politiques publiques (RGPP) - 2007-2012, Siècles, 35-36. http://journals.openedition.org./siecles/1436

Gélédan, F. (2016). Spectres du Léviathan. L’État à l'épreuve de la simplification administrative (2006-2015), Revue française d'administration publique, 1 (157), 33-48.

Gélédan, F. (2019). Les cabinets de conseil dans la Révision générale des politiques publiques (RGPP) : une importation sous contrainte. Dans Matyjasik, N. \& Genoun, M. (dir.), En finir avec le New Public Management, Vincennes : Presses de l'Institut de la gestion publique et du développement économique.

Hood, G. (2000). The Art of the state: Culture, rhetoric, and public management. Oxford : Oxford University Press.

IHL, O., Kaluszynski, M. \& Pollet, G. (dir.) (2003). Les Sciences de gouvernement. Paris : Economica.

Ihl, O. (dir.) (2006). Les « Sciences» de l'action publique. Grenoble : Presses universitaires de Grenoble. 
Ingold, A. (2011). Cartographier pour naturaliser au XIX ${ }^{\mathrm{e}}$ siècle. Les ingénieurs des mines et la carte hydrographique d'Italie. Dans Laborier, P., Audren, F., Napoli, P. \& Vogel, J. (dir.), Les Sciences camérales : activités pratiques et histoire des dispositifs publics (p. 539 567). Paris : Presses universitaires de France.

Jeannot, G. (2006). Des technologies d'enquête participative dans le développement rural. Dans Ihl, O. (dir.), Les « Sciences » de l'action publique (p. 219-231). Grenoble : Presses universitaires de Grenoble.

Laborier, P., Audren, F., Napoli, P. \& Vogel, J. (dir.) (201 1). Les Sciences camérales : activités pratiques et histoire des dispositifs publics. Paris: Presses universitaires de France

Le Bourhis, J.-P. (2003). Quadriller le territoire. La cartographie au service de l'action publique contre les risques naturels. Dans Ihl, O., Kaluszynski, M. \& Pollet, G. (dir.), Les Sciences de gouvernement (p. 157-167). Paris : Economica.

Linhardt, D. \& Moreau de Bellaing, C. (2017). La doctrine du droit pénal de l'ennemi et l'idée de l'antiterrorisme. Genèse et circulation d'une entreprise de dogmatique juridique. Droit et société, 97 (3), 615-640.

Mitchell, T. (1988). Colonizing Egypt. Berkeley: University of California Press.

Overath, P. (2011). Entre interprétation de crises et scénarios de guerre. Les représentations de la politique de la population en France et en Allemagne (1870-1918). Dans Laborier, P., Audren, F., Napoli, P. \& Vogel, J. (dir.), Les Sciences camérales : activités pratiques et histoire des dispositifs publics (p. 421-435). Paris : Presses universitaires de France.

Potin, Y. (2000). L'État et son Trésor. Actes de la recherche en sciences sociales, (133), 48-52.

Retière, J.-N. (2003). L’ingénieur et le préfet. Prévoir la crue de la Loire (1846-1936). Dans Ihl, O., Kaluszynski, M. \& Pollet, G. (dir.), Les Sciences de gouvernement (p. 169-179). Paris : Economica.

Scott, J. C. (1998). Seeing like a state: How certain schemes to improve the human condition have failed. New Haven : Yale University Press.

Shapin, S. \& Schaffer, S. (1985). Le Léviathan et la pompe à air. Hobbes et Boyle entre science et politique (traduit par T. Piélat). Paris : La Découverte.

Tanguy, G. (2011). Les préfets face à la grève : faire savoir, savoir-faire et « expertise de gouvernement ». Dans Laborier, P., Audren, F., Napoli, P. \& Vogel, J. (dir.), Les Sciences camérales: activités pratiques et histoire des dispositifs publics (p. 437-465). Paris: Presses universitaires de France.

Vial, S. (2015). Qu'est-ce que la recherche en design? Introduction aux sciences du design. Sciences du design, 1 (1), 22-36.

Vogel, J. (2011). Les mines dans les pays germaniques et en France aux XVIIIe et XIX ${ }^{\text {e }}$ siècles. Genèses et frontières d'une expertise scientifique et administrative. Dans Laborier, P., Audren, F., Napoli, P. \& Vogel, J. (dir.), Les Sciences camérales: activités pratiques et histoire des dispositifs publics (p. 399-419). Paris : Presses universitaires de France.

Weller, J.-M. (1999). L'État au guichet. Sociologie cognitive du travail et modernisation administrative des services publics. Paris : Desclée de Brouwer. 
Marie Alauzen et Coline Malivel. Le design est-il en passe de devenir une science de gouvernement ? Réflexion sur les espoirs suscités par les sciences du design dans la modernisation de l'État en France (2014-2019), Sciences du Design, 12 (2), 2020, pp. 36-47. DOI: 10.3917/sdd.012.0036.

Weller, J.-M. \& Pallez, F. (2017). Les formes d'innovation publique par le design : un essai de cartographie, Sciences du Design, 1 (5), 32-5.

Marie Alauzen est attachée temporaire d'enseignement et de recherche en science politique à
l'École normale supérieure et chercheuse associée au Centre Maurice Halbwachs ÉHESS-
ÉNS (UMR CNRS 8097) et au Centre de sociologie de l'innovation de l'École des mines de
Paris (UMRCNRS I3 9217).
marie.alauzen@ens.pls.eu
Coline Malivel est designeuse à Étalab, rattachée à la Direction interministérielle du
$\begin{aligned} & \text { numérique(DiNUM). } \\ & \text { coline.malivel@gmail.com }\end{aligned}$

